\title{
Occupational risk of human Cytomegalovirus and Parvovirus B19 infection in female day care personnel in the Netherlands; a study based on seroprevalence
}

\author{
F. F. Stelma • A. Smismans • V. J. Goossens • \\ C. A. Bruggeman - C. J. P. A. Hoebe
}

Received: 23 May 2008 / Accepted: 13 September 2008 / Published online: 11 October 2008

(C) The Author(s) 2008. This article is published with open access at Springerlink.com

\begin{abstract}
Cytomegalovirus (CMV) and Parvovirus B19 infections acquired during pregnancy may result in developmental disabilities of the foetus. This study evaluates the occupational risk of these infections in female day care personnel. IgG seroprevalence was determined in 310 Dutch day care workers and 158 nursing school students. CMV seroprevalence was age-related, starting at $21 \%$ in those $<20$ years and reaching $65 \%$ in those $>35$ years. Between the ages of 20 and 24 years the CMV prevalence was higher in day care personnel than in controls, $50 \%$ versus $31 \%(p=0.03)$. In the first 2 years of employment the risk of attracting CMV was significantly increased $\left(\mathrm{OR}_{\mathrm{adj}}=3.80 ; p<0.001\right)$ and the occupational risk was also increased $\left(\mathrm{OR}_{\mathrm{adj}} 2.19 ; p<0.001\right)$. Parvovirus seropositivity $(71-77 \%)$ was not related to age or working at a day care centre. In conclusion, an occupational risk was observed for CMV, but not for Parvovirus infection in female day care personnel.
\end{abstract}

The fieldwork was performed at the Department of Infectious Diseases, South Limburg Public Health Service. Analyses and editing were finalised at the Department of Medical Microbiology, University Hospital Maastricht.

F. F. Stelma $(\bowtie) \cdot$ A. Smismans $\cdot$ V. J. Goossens $\cdot$

C. A. Bruggeman $\cdot$ C. J. P. A. Hoebe

Department Medical Microbiology,

University Hospital Maastricht,

P.O. Box 5800, 6202 AZ Maastricht, The Netherlands

e-mail: fstel@lmib.azm.nl

C. J. P. A. Hoebe

Department of Infectious Diseases,

South Limburg Public Health Service,

Geleen, The Netherlands

\section{Introduction}

Infections with Parvovirus B19-the causative agent of erythema infectiosum or fifth disease - and Cytomegalovirus (CMV) are global and common. Both infections have a benign or asymptomatic course in the immunocompetent host, while in the immunocompromised patient the viruses can severely affect health. Another important risk group for CMV and Parvovirus infections are pregnant women, with a reported incidence that varies between 0.09 and $2 \%$ and 1 and $5 \%$ respectively $[1,2]$. Since few newborns are screened for CMV and Parvovirus, the true impact of congenital CMV and Parvovirus infection is unknown. Intrauterine transmission of CMV occurs in approximately $40 \%$ of the primary CMV infections [3, 4]. Ten percent of these infected infants present at birth with symptoms of irreversible central nervous system involvement including microcephaly, encephalitis, seizures, deafness, upper motor neuron disease and mental retardation. In addition, $10-17 \%$ of the infants that are asymptomatic at birth develop sensorineural hearing loss or neurodevelopmental sequelae months to years afterwards [5, 6]. Transplacental transmission rates of Parvovirus have been estimated to be between 25 and $33 \%$. Fetal infection with Parvovirus may lead to severe anemia, generalised edema, congestive heart failure and myocarditis, leading to fetal death in $5-9 \%$ of the cases, with the greatest risk in the second trimester of pregnancy.

Approximately 60 and $30 \%$ of the women of childbearing age in developed western European countries, such as the Netherlands, are still susceptible to developing either a primary CMV or Parvovirus infection [7, 8]. Nevertheless, there are great variations in seroprevalence according to geographical region, socioeconomic status and ethnic composition [5]. Therefore, it is important to establish local sero-epidemiology data to offer preventive strategies in a 
given country, as CMV and Parvovirus infections cannot yet be prevented by immunisation and therapeutic options are questionable. In the Netherlands, in a recent prospective study, an unusually low incidence of congenital CMV infection of $0.09 \%$ was reported [7]. The authors concluded that preventive measures for CMV were thus not justified, and that antenatal and perinatal counselling should be reserved for (upcoming) Parvovirus epidemics. However, this incidence is based on calculations in the general population and small, potentially high-risk groups such as day care workers will not be identified. Young children are an important source of CMV or Parvovirus infection due to poor hygiene. Up to $71 \%$ of children attending day care centres may carry CMV in saliva or urine [9]. Direct and indirect exposure of day care personnel by touching, diapering, washing, feeding and contact with environmental surfaces put them at a higher risk of acquiring a viral infection $[9,10]$. The aim of our study was to assess the occupational risk of CMV and Parvovirus B19 infection in female day care personnel in the Netherlands to determine whether or not preventive measures (for instance, serological testing followed by alternative work during pregnancy being offered to women who test $\mathrm{CMV}$-seronegative) should be offered or not.

\section{Materials and methods}

\section{Participants}

Cytomegalovirus and Parvovirus IgG seroprevalence was determined in 310 sera collected from 313 female day care personnel from 66 regional day care facilities for children in the southern part of the Netherlands between October 2000 and April 2003. Due to laboratory-related factors, 3 samples were not tested for CMV and Parvovirus. Written consent to participation was obtained from all participants. All women were asked to complete a questionnaire, including data regarding age, work seniority, infant ages of the care group, and the number and age of their own children at home. Data were entered into a codified database for analysis. One hundred and fifty-eight female students, aged between 17 and 26 years, attending a nurse school in the same region as the day care centres, served as a control group. Data on their age and own children at home were obtained.

\section{Laboratory analysis}

The outcome measure was seroprevalence for CMV and Parvovirus infection. Sera were frozen at $-20^{\circ} \mathrm{C}$ until testing for CMV and Parvovirus IgG. CMV IgG was determined by an automated microparticle enzymatic immunoassay AxSym
CMV IgG version (Abbott laboratories, Abbott Park, IL, USA) and Parvovirus $\operatorname{IgG}$ was assayed using a third generation Parvovirus IgG enzyme-linked immunoassay (Biotrin, Dublin, Ireland). Both assays were performed according to the manufacturer's instructions. A value of $\geq 15 \mathrm{U} / \mathrm{ml}$ for CMV IgG was considered positive, whereas a ratio of optical density sample to optical density cut-off $(\mathrm{S} / \mathrm{CO})$ of more than 1 was considered positive for Parvovirus $\operatorname{IgG}$.

\section{Statistical analysis}

Proportions between the groups were compared using Chisquared statistics. Associations were tested by univariate and multiple logistic regression analysis, the latter allowing adjustment for co-variables. The outcome variable of the regression models was CMV IgG seropositivity versus seronegativity. Parvovirus B19 was not considered in a regression model as no interesting age-related features and differences between groups were observed during the explorative Chi-squared analyses. Two different regression models were tested (Table 1). The first model tested the main effect "occupational risk," defined as being a day care worker or student (= control). The second model tested the main effect "work seniority" as defined by five categories reflecting the number of years of day care employment (0 years, $1-2$ years, 3-4 years, $5-9$ years, $>9$ years). Covariables in both models were "age" in years, "having own children" as defined by parity (no children versus $\geq 1$ child) and "having children who were in contact with other children" as defined by the presence of children at home attending school or a day care centre (yes versus no). All co-variables were simultaneously included in the logistic regression model.

\section{Results and discussion}

The main finding of this study was that female day care personnel have an increased risk of attracting a primoinfection with $\mathrm{CMV}\left(\mathrm{OR}_{\mathrm{adj}} 2.19, p<0.001\right.$; Table 1), especially during the first 2 years of day care employment $\left(\mathrm{OR}_{\text {adj }}\right.$ 3.80, $p<0.001$; Table 1$)$. Furthermore, an agedependent increase in CMV IgG seropositivity was observed for both day care workers and controls (Fig. 1a). Of women younger than 19 years, $12.5 \%$ (1 out of 8 ) and $22 \%$ (23 out of 104) respectively were positive for CMV IgG. Fifty percent ( 40 out of 80 ) and $31 \%$ (16 out of $52 ; p=0.03$ ) of women aged between 21 and 24 years and 53\% (31 out of 58 ) and $50 \%$ ( 1 out of 2 ) of women aged between 25 and 29 years presented as CMV IgG-seropositive respectively. In day care personnel aged between 30 and 34 years, 59\% (30 out of 51), and in the age group above 35 years, $65 \%$ (73 out of 113) were CMV IgG-seropositive. No women 
Table 1 Cytomegalovirus (CMV) seroprevalence in female day care personnel $(n=310)$ and controls $(n=158)$ and odds ratios calculated by logistic regression analysis in female day care workers and controls

\begin{tabular}{|c|c|c|c|c|c|c|c|}
\hline & & Total number ${ }^{\mathrm{a}}$ & Positive IgG CMV number (\%) & $\mathrm{OR}_{\text {crude }}$ & $95 \% \mathrm{CI}$ & $\mathrm{OR}_{\text {adj }}$ & $95 \% \mathrm{CI}$ \\
\hline \multirow[t]{10}{*}{ Model 1} & \multicolumn{7}{|l|}{ Occupational risk } \\
\hline & Control & 158 & $40(25)$ & 1 & & 1 & \\
\hline & Day care personnel & 310 & $175(57)$ & 3.82 & $2.51-5.84$ & 2.19 & $1.28-3.74$ \\
\hline & \multicolumn{7}{|c|}{ Parity; number of own children } \\
\hline & 0 & 302 & $111(37)$ & 1 & & 1 & \\
\hline & $\geq 1$ & 160 & $99(62)$ & 2.79 & $1.88-4.15$ & 0.76 & $0.34-1.66$ \\
\hline & \multicolumn{7}{|c|}{ Children in day care or at school } \\
\hline & No & 371 & $152(41)$ & 1 & & 1 & \\
\hline & Yes & 97 & $63(65)$ & 2.67 & $1.68-4.25$ & 1.70 & $0.86-3.36$ \\
\hline & Age (years) & & & - & & 0.96 & $0.93-0.99$ \\
\hline \multirow[t]{13}{*}{ Model 2} & \multicolumn{7}{|l|}{ Work seniority (years) } \\
\hline & 0 & 158 & $40(25)$ & 1 & & 1 & \\
\hline & $1-2$ & 78 & $36(46)$ & 2.53 & $1.43-4.48$ & 3.80 & $1.53-9.38$ \\
\hline & $3-4$ & 69 & $32(46)$ & 2.55 & $1.41-4.62$ & 2.06 & $0.93-4.58$ \\
\hline & $5-9$ & 90 & $57(63)$ & 5.10 & $2.91-8.91$ & 2.04 & $0.93-4.50$ \\
\hline & $>9$ & 70 & $48(69)$ & 6.44 & $3.47-11.95$ & 1.06 & $0.52-2.19$ \\
\hline & \multicolumn{7}{|c|}{ Parity; number of own children } \\
\hline & 0 & 302 & $111(37)$ & 1 & & 1 & \\
\hline & $\geq 1$ & 160 & $99(62)$ & 2.79 & $1.88-4.15$ & 0.69 & $0.31-1.54$ \\
\hline & \multicolumn{7}{|c|}{ Children in day care or at school } \\
\hline & No & 371 & $152(41)$ & 1 & & 1 & \\
\hline & Yes & 97 & $63(65)$ & 2.67 & $1.68-4.25$ & 1.83 & $0.91-3.67$ \\
\hline & Age (years) & & & - & & 0.97 & $0.94-1.01$ \\
\hline
\end{tabular}

$\mathrm{OR}=$ odds ratio $; 95 \% \mathrm{CI}=95 \%$ confidence interval of odds ratio; $\mathrm{OR}_{\text {crude }}=$ crude odds ratio; $\mathrm{OR}_{\mathrm{adj}}=$ adjusted odds ratio

${ }^{a}$ Total group $N=468$, discrepant numbers are due to missing values

older than 26 years were encountered in the control group. Therefore, CMV seroprevalence data above this age are not available in this group, which is a limitation of our study. Age-dependent CMV seroprevalence has been described before, with an annual seroconversion rate of $1 \%$ throughout adulthood [3]. Therefore, similar age groups to those of the day care personnel and the control group were compared. In both study populations, the expected agedependent increase in CMV seroprevalence was observed; however, this increase was greater in day care personnel under 25 years of age than in the control population. The fact that day care personnel are more likely to be exposed to a CMV infection is additionally supported by the ageadjusted associations between CMV seropositivity and day care workers' seniority found in the present study.

Work seniority was positively associated with an increased risk of becoming CMV-seropositive (see also Table 1), but when adjusting for age, having children and children in day care or at school, only the first 2 years of day care employment showed any significant influence. In day care workers, CMV seroprevalence was associated with having children at home (OR 2.79, $p<0.001$; Table 1) and with having children attending a day care centre or a primary school (OR 2.67, $p<0.001$; Table 1). However,
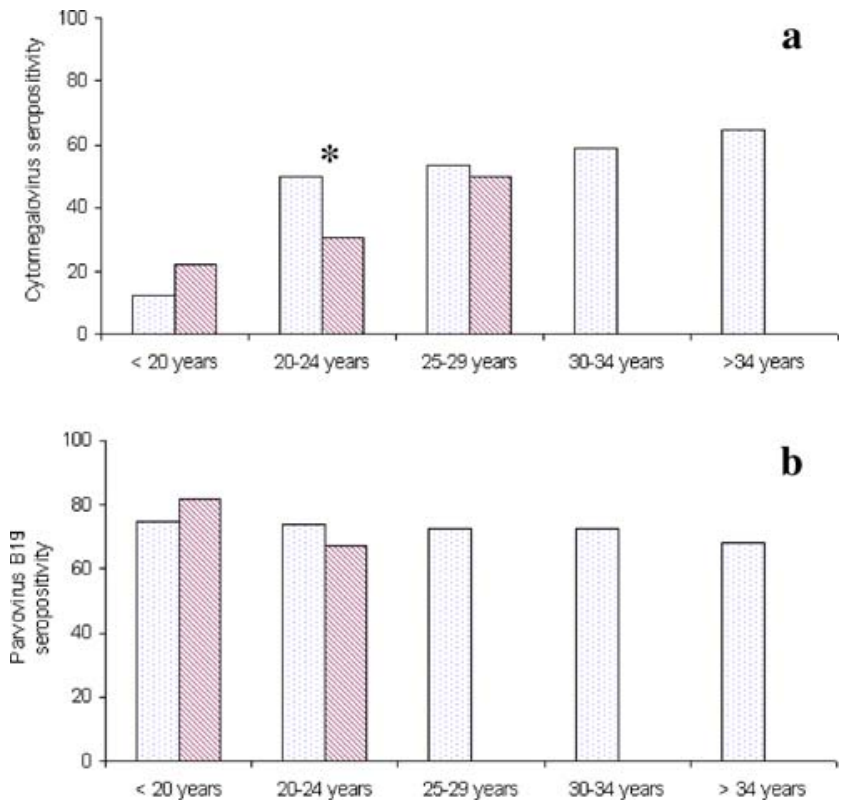

Fig. 1 a Age-related distribution of Cytomegalovirus seropositivity in female day care personnel and controls. In the age group 20-24 years the CMV seropositivity was significantly higher in day care personnel than in controls ( 50 versus $31 \%$; $p=0.03 *$ ). b Age-related distribution of Parvovirus B12 seropositivity in female day care personnel and controls. No relation was seen with regard to age and no difference between day care personnel and controls was observed 
when adjusting for co-variables, these associations were no longer significant (see Table 1).

Parvovirus serology was performed in both study groups. No clear age-related increase in positive serology was observed in either group (see Fig. 1b). Also, no overall significant difference was seen between the two groups, as the seroprevalence was $71 \%(n=319)$ and $77 \%(n=158)$ respectively $(p=0.224$; Fig. $1 b)$. The mean Parvovirus seroprevalence of about $70 \%$ was comparable with the seroprevalence of $70 \%$ measured in the Netherlands [8]. Parvovirus B19 is a highly contagious viral disease causing erythema contagiosum, which is transmitted by droplets and aerosols. Due to the general mode of transmission, all young members of the general population are at increased risk of acquiring the infection. According to a recent study in the UK, antibody prevalence rose nonlinearly with age from $21 \%$ in children aged $1-4$ years to $>75 \%$ in adults aged $\geq 5$ years, with the highest risk of acquiring infection being in those aged $<15$ years [11]. Therefore, it is not surprising that there is no occupational risk associated with Parvovirus B19.

The main limitation of our study was the fact that the control group is different from the day care workers; it had a limited age range (17-26 years) and the female students mainly had no own children or exposure to young children. The main purpose of the control group was therefore to establish the occupational risk of CMV and Parvovirus B19 infection adjusted for age.

Our data are important as CMV is not considered a public health problem in the Netherlands, whereas other countries, such as the US, recognise that women within certain professions like day care are more at risk of acquiring a primary CMV infection during pregnancy [9, 10]. The rate of CMV IgG seropositivity found in our control group mirrors the $35 \%$ observed in an earlier Dutch study performed by Gaytant and coworkers [7]. Sixty-five percent of Dutch women of childbearing age are thus still at risk of attracting a CMV primo-infection. Nevertheless, there appears to be little CMV-related morbidity in children in the Netherlands. In a recent Dutch study performed in the metropolitan agglomeration formed by the cities Amsterdam, Rotterdam and The Hague, CMV seropositive neonates were followed for 24 months. No significant symptoms of CMV infection were observed in these infants [7]. From studies outside the Netherlands it is known that about $7-15 \%$ of asymptomatic infants will eventually develop sensorineural hearing loss [5]. However, the CMV seroprevalence in the Netherlands is lower than that in the United States and other western European countries, ranging from 40 to $83 \%$ [5]. Studies from other countries have demonstrated that women working at child-care centres are at increased risk of acquiring primo-CMV infections [10], and our study confirms that this is also the case in the Netherlands.
Cytomegalovirus infection, is mediated through contact with infant saliva and urine, exposure mainly associated with caring for young infants. Children who attend day care centres are at a higher risk of acquiring infections due to close contact and the sharing of toys and food. In addition, infected children may excrete CMV for a prolonged time. According to different reports, up to $50 \%$ of children attending day care centres may excrete CMV [9].

Preconceptional maternal immunity (i.e., IgG seropositivity) has been demonstrated to protect against congenital CMV [12, 13]; however, several studies have also pointed out that recurrent $\mathrm{CMV}$ infections, as well as reactivation of latent infections, may cause congenital CMV [12]. Our data neglect the possibility of a recurrent $\mathrm{CMV}$ infection and the true occupational risk of CMV infections will therefore be even higher.

Hitherto, the only mode of prevention for congenital CMV disease has consisted of increasing population awareness and improvement of hygienic practices in those at increased risk. The availability of a licensed and effective CMV vaccine will probably take years and drug treatment for pregnant women has not yet been extensively studied because of concerns about toxicity. In a recent study, CMV hyperimmune globulins were administered to CMVinfected pregnant women [14]. Although the results suggested that passive immunisation could prevent congenital CMV infection, the results should be further substantiated in a randomised and controlled trial. As such, women in contact with small children, or those who are planning a pregnancy, at least deserve to be informed how to prevent CMV acquisition through improved personal hygiene [15]. Also, serological screening of pregnant women who are at increased risk, followed by offering alternative duties at work in those tested negative, should be considered.

Open Access This article is distributed under the terms of the Creative Commons Attribution Noncommercial License which permits any noncommercial use, distribution, and reproduction in any medium, provided the original author(s) and source are credited.

\section{References}

1. Ergaz Z, Ornoy A (2006) Parvovirus B19 in pregnancy. Reprod Toxicol 21(4):421-435 doi:10.1016/j.reprotox.2005.01.006

2. Gaytant MA, Steegers EA, Semmekrot BA et al (2002) Congenital cytomegalovirus infection: review of the epidemiology and outcome. Obstet Gynecol Surv 57(4):245-256 doi:10.1097/ 00006254-200204000-00024

3. Griffiths PD, Baboonian C (1984) A prospective study of primary cytomegalovirus infection during pregnancy: final report. $\mathrm{Br} \mathrm{J}$ Obstet Gynaecol 91(4):307-315

4. Peckham CS (1991) Cytomegalovirus infection: congenital and neonatal disease. Scand J Infect Dis Supp1 80:82-87

5. Griffiths PD, Walter S (2005) Cytomegalovirus. Curr Opin Infect Dis 18(3):241-245 doi:10.1097/01.qco.0000168385.39390.1b 
6. Williamson WD, Demmler GJ, Percy AK et al (1992) Progressive hearing loss in infants with asymptomatic congenital cytomegalovirus infection. Pediatrics 90(6):862-866

7. Gaytant MA, Galama JM, Semmekrot BA et al (2005) The incidence of congenital cytomegalovirus infections in The Netherlands. J Med Virol 76(1):71-75 doi:10.1002/jmv.20325

8. Zaaijer HL, Koppelman MH, Farrington CP (2004) Parvovirus B19 viraemia in Dutch blood donors. Epidemiol Infect 132 (6):1161-1166 doi:10.1017/S0950268804002730

9. De Mello AL, Ferreira EC, Vilas Boas LS et al (1996) Cytomegalovirus infection in a day-care center in the municipality of Sao Paulo. Rev Inst Med Trop Sao Paulo 38(3):165-169 doi:10.1590/S0036-46651996000300001

10. Murph JR, Baron JC, Brown CK et al (1991) The occupational risk of cytomegalovirus infection among day-care providers. JAMA 265(5):603-608 doi:10.1001/jama.265.5.603
11. Vyse AJ, Andrews NJ, Hesketh LM et al (2007) The burden of parvovirus B19 infection in women of childbearing age in England and Wales. Epidemiol Infect 135:1354-1362

12. Fowler KB, Stagno S, Pass RF (2003) Maternal immunity and prevention of congenital cytomegalovirus infection. JAMA 289 (8):1008-1011 doi:10.1001/jama.289.8.1008

13. Gaytant MA, Rours GI, Steegers EA et al (2003) Congenital cytomegalovirus infection after recurrent infection: case reports and review of the literature. Eur J Pediatr 162(4):248-253

14. Nigro G, Adler SP, La Torre R et al (2005) Passive immunization during pregnancy for congenital cytomegalovirus infection. N Engl J Med 353(13):1350-1362 doi:10.1056/ NEJMoa043337

15. Jeon J, Victor M, Adler SP et al (2006) Knowledge and awareness of congenital cytomegalovirus among women. Infect Dis Obstet Gynecol 14(3):80383 